\title{
Stadtstaaten oder Barbarei?
}

\author{
(Anti-)Urbanität, Demographie und munizipale Perspektiven[1]
}

Tobias Bernet

Der vorliegende Essay betrachtet den aktuellen Zulauf zu rechten Politikangeboten aus Sicht einer kritischen Stadtforschung. Mit Blick auf demographische Prozesse - insbesondere Binnenmigration - reflektiert er die These, dass rechte Ideen und Gruppierungen vor allem in ländlichen ,abgehängten Gebieten' eine hohe Zustimmung finden, während als ,kosmopolitisch' und ,modernisierungsbefürwortend' beschriebene großstädtische Lebenswelten dafür weniger empfänglich seien. Unter Bezugnahme auf deutsche wie amerikanische Debatten wird dafür plädiert, die Verräumlichung politischer Einstellung stärker dynamisch und relational zu konzeptualisieren und ein Augenmerk auf kleinräumige Differenzierungen innerhalb von Städten und Regionen zu richten. Damit gerät das emanzipatorische Potential des Munizipalen als politische Bezugsgröße jenseits des Nationalstaates in den Blick."

Ersteinreichung: 14. Oktober 2018; Veröffentlichung online: 15. Mai 2019

An English abstract can be found at the end of the document.

\section{We gotta get out while we're young: Einleitung}

Baby, this town rips the bones from your back

It's a death trap, it's a suicide rap

We gotta get out while we're young

'cause tramps like us, baby we were born to run

Bruce Springsteen, „Born to Run“ (1975)

Hat eigentlich mal jemand Bruce Springsteen zugehört? Der Sänger aus New Jersey ist eine kulturelle Ikone jenes Amerika, das man sich kleinstädtisch bis ländlich denkt, patriarchal und eher weiß, blue-collar-proletarisch und vor allem postindustriell verheert, ,abgehängt'. Und das aus Sicht soziologischer Beobachter_innen und journalistischer Kommentator_innen in seinen politischen Präferenzen spätestens seit der Wahl Donald Trumps extrem erklärungsbedürftig erscheint. Ein ganzes Genre hat sich in den letzten Jahren herausgebildet, das Trump-Wähler_innen ebenso wie Anhänger_innen der AfD, Brexit-Befürworter_innen und die ,besorgten Bürger_innen' anderer Länder nicht nur zu erklären, sondern auch zu verstehen sucht. Die dabei oftmals als Patentrezept deklarierte Praxis des ,Ernstnehmens lassen zahlreiche Autor_innen jedoch nicht in erster Linie diesen Menschen zuteilwerden, sondern - sie selbst gleichsam infantilisierend - den von ihnen 
geäußerten Sorgen. Doch wer schon einmal Sorgen hatte, sollte wissen, dass man diese selbst nicht unbedingt am akkuratesten erfasst. Daher geht die hier folgende Auslotung der Untiefen solcher Analysen davon aus, dass zu einem demokratischen Diskurs nicht die Abwertung der oder des Andersdenkenden gehört, wohl aber engagierter Widerspruch gegen menschenfeindliche und irrationale Äußerungen - gerade um auch jene, die solche tätigen, als prinzipiell vernunftbegabte Menschen, eben: ernst zu nehmen.

Eher noch als die von möglicherweise nur halb verstandenen Sorgen Befallenen selbst tut der Barde Wahrheit kund. Springsteens Interesse galt schon immer eher den Schattenseiten des amerikanischen Traums. 1984 wehrte er sich gegen die Vereinnahmung von „Born in the USA“ durch Ronald Reagan, seither hat er sich immer wieder explizit gegen republikanische Politiker_innen positioniert, zuletzt auch gegen Trump. Und schon in „Born to Run“ besang er gerade nicht die vermeintliche Idylle der Zwangsvergemeinschaftung in den amerikanischen middletowns, sondern im Gegenteil: das Abhauen in die Großstadt. Dorthin wo es mehr ökonomische und persönliche Entfaltungsmöglichkeiten gibt, mehr Freiheit, mehr Rock'n'Roll - und damit auch mehr diversity, zumal beim Erscheinen des Songs die epochale Vitalisierung der populären Musik durch den schwarzen Sound noch keine dreißig Jahre zurücklag.

Dieser Empfehlung sind die Leute in Massen gefolgt - im amerikanischen rustbelt, in den nordfranzösischen Industrieregionen, in ganz Ostdeutschland außerhalb der Groß- und Universitätsstädte. Wenn die Menschen in den Hochburgen der US-Republikaner_innen, des Front (neu „Rassemblement“) National oder der AfD meinen, Probleme zu haben, die durch Migrationsbewegungen verursacht sind, so haben sie folglich in gewisser Weise nicht unrecht - und irren sich doch um 180 Grad. Die Abwanderung ist das Schicksal dieser Orte, die Zuwanderung dorthin in aller Regel vernachlässigbar. Auch die solchen Regionen gegebenenfalls zugewiesenen Geflüchteten ziehen mehrheitlich in die Großstädte, sobald es ihnen rechtlich und wirtschaftlich möglich ist. Bei aller Ablehnung von Neuankömmlingen mag dies auch als narzisstische Kränkung empfunden werden.

Mit Blick auf solche Verräumlichungsprozesse soll im Folgenden ein Versuch unternommen werden, den Aufschwung und Wandel rechter Politikangebote während der letzten Jahre von der Warte einer kritischen Stadt- und Raumforschung aus zu reflektieren. Ausgangspunkt ist der gerade anlässlich von Wahlergebnissen immer wieder betonte Gegensatz zwischen liberalen bis linken Großstädten und dem konservativen bis rechten ländlichen und kleinstädtischen Raum. Rechte Politikangebote erreichen demnach unter jenen hohe Zustimmungswerte, die - geographisch und/oder ideell abseits von ,kosmopolitisch ' und ,modernisierungsbefürwortend' gedachten großstädtischen Lebenswelten zuhause sind.

Ausgehend von einer Einordnung dieser Erklärungsschemata betrachtet dieser Beitrag den postulierten Stadt-Land-Gegensatz mit Blick auf demographische Prozesse - insbesondere Binnenmigration - stärker dynamisch und relational. Vieles deutet nämlich darauf hin, dass die politischen Bruchlinien oft nicht einfach zwischen städtischen und ländlichen, sondern zwischen wachsenden und schrumpfenden Gebieten verlaufen. Das heißt: Die Art und Weise, wie ,kosmopolitische‘ Urbanität realiter entsteht, 
produziert ihr Anderes, die ,abgehängten Regionen', stets mit. Mit Blick darauf, dass dieser „demographischen Polarisierung“ mittlerweile - in Deutschland wie anderswo - „das Potential, die Demokratie zu unterminieren“(Müller 2018) zugesprochen wird, soll hier über mögliche Neufassungen politischer Bezugsgrößen nachgedacht werden: Ist die grobe Maßstäblichkeit von Nationalstaaten der Komplexität individueller Lebensführungen und globaler Problemstellungen im 21. Jahrhunderts noch angemessen? Oder ist das Ideal munizipaler Selbstverwaltung womöglich das adäquatere demokratische Gegenstück zur mafiös-neofeudalen Selbstherrlichkeit, die der Grundrentenkapitalist Trump verkörpert?

\section{Wieso haben die Rechten Erfolg?}

Die räumlich-physische Metapher der ,Abgehängtheit‘ scheint in gewisser Weise alle zur Zeit verbreiteten Erklärungsansätze für den Erfolg rechter politischer Kräfte zu durchdringen, wobei zunächst zwei Hauptrichtungen unterschieden werden können: Die eine verweist vor allem auf soziale Benachteiligung, deutet Rechtswähler_innen also in erster Linie als vom neoliberalen Status quo Enttäuschte (vgl. z. B. Vehrkamp/Wegschaider 2017); die andere hebt eher ,kulturelle‘ Aspekte hervor - namentlich die Tatsache, dass die letztlich rassistischen Einstellungen, die etwa den Erfolg der AfD befeuern, auch unter Angehörigen mittlerer und hoher Einkommens- und Statusgruppen verbreitet sind (vgl. z. B. Lengfeld 2017a).[2]

Doch selbstredend gedeihen Einstellungen nicht im luftleeren Raum. Rassistische - allgemeiner: ,modernisierungsfeindliche - Orientierungen finden an manchen Orten sehr viel mehr Zuspruch als an anderen. Damit erscheinen vor allem Erklärungsansätze interessant, die räumliche Aspekte hervorheben und dabei ökonomische Faktoren ebenso einbeziehen wie kulturelle. Im deutschen Kontext gehen derartige Erörterungen zumeist mit einem Blick auf die Eigenheiten der neuen Bundesländer einher - wo die AfD in ländlichen und städtischen Räumen im Vergleich mit westdeutschen Pendants durchschnittlich besser abschneidet. Die Erklärungen dafür sind wiederum vielfältig. Manche beschreiben eine Kontinuität der Dominanz von in der DDR wohlgelittenen, preußischen', letztlich gar bis zum Dreißigährigen Krieg zurückreichenden autoritären Ideenwelten (Brechtken 2017, als Replik darauf Asch 2018, vgl. auch Cantoni/Hagemeister/Westcott 2019). Öfter wird jedoch auf die Erfahrungen der Nachwendezeit verwiesen, insbesondere auf eine wahrgenommene Abwertung von Biographien und Deutungshorizonten, die manche Ostdeutsche - auch Jahre später - als einen Mangel an Anerkennung insbesondere im Vergleich mit Migrant_innen bewerten. „Integriert doch erst mal uns“, bekam die sächsische Staatsministerin für Gleichstellung und Integration Petra Köpping in Gesprächen angeblich immer wieder zu hören (Köpping 2018, zur Vergleichbarkeit der Stellung von Ostdeutschen und Menschen mit Migrationshintergrund in der wiedervereinigten Bundesrepublik vgl. unter anderem Foroutan/Kubiak 2018).

Andere Beiträge heben mit Blick auf die Nachwendezeit vor allem die Erfahrung der ,Erfolge ' gewaltförmiger rassistischer Mobilisierungen hervor: Dass pogromartige Ausschreitungen in Hoyerswerda, Rostock und anderswo kaum sanktioniert, durch die 1992 beschlossenen Einschränkungen des 
Asylrechts in gewisser Weise gar honoriert wurden, habe zu einer Legitimierung und Perpetuierung gewaltaffiner rechter Einstellung in Teilen der ostdeutschen Gesellschaft geführt (Hensel 2017, Begrich 2018). Es sind Milieus entstanden, die Gewalt gegenüber ,Feinden' und ,Anderen' - bis hin zu den terroristischen Morden des NSU - gutheißen oder zumindest hinnehmen. Dass dabei auch Vorstellungen patriarchaler Männlichkeit eine wichtige Rolle spielen, wird in Ostdeutschland ebenfalls durch räumliche Aspekte akzentuiert: Da Frauen aus ländlichen Gebieten in deutlich höherer Zahl abwandern als Männer, entstehen „Regionen mit Männerüberschuss“, in denen „die soziale Kälte Einzug hält“ (Koester 2018). Dies übersetzt sich wiederum in hohe Zustimmungswerte für die AfD, die von wesentlich mehr Männern als Frauen gewählt wird.

Rechte Kräfte sind jedoch auch in Westdeutschland (vgl. Franz/Fratzscher/Kritikos 2018, Bergmann/Diermeier/Niehues 2018) und in anderen Ländern in dünn besiedelten, von Bevölkerungsschwund betroffenen und damit überalterten Räumen besonders erfolgreich - was letztlich jedem auf ostdeutsche Besonderheiten abhebenden Erklärungsversuch Grenzen setzt. Die „Rache der Peripherie“ (Rada 2017, vgl. auch Kaschuba 2016) ist auch in Polen, Österreich, Frankreich oder den USA zu beobachten. Man wird der Komplexität des Phänomens also eher vermittels einer transnationalen Perspektive auf Stadt-Land-Gegensätze auf die Spur kommen.

\section{Gräben und Schwärme:}

\section{Die Polarisierung räumlicher Entwicklungen}

Der grundlegende Befund fällt bei der Betrachtung der nach Wahlen jeweils veröffentlichten Karten, auf denen Stimmbezirke nach Parteipräferenzen eingefärbt sind, sofort ins Auge: Städte wählen linker und liberaler, ländliche Gebiete rechter und konservativer.[3] Relevant für die Deutung aktueller politischer Entwicklungen ist dieses Phänomen vor allem deswegen, weil es sich nachweislich in den letzten Jahrzehnten verstärkt hat. Eine entsprechende quantitative Untersuchung wurde unlängst etwa für die Schweiz vorgelegt. Diese hatte lange Zeit ihren eigenen Ost-West-Gegensatz: den sogenannten ,Röstigraben“ zwischen den Sprachregionen. Auch dieser wird jedoch zunehmend durch einen Stadt-Land-Gegensatz abgelöst, wie Shin Alexandre Koseki (2017) nachweisen konnte. Er analysierte die in der Schweiz üblichen landesweiten Volksabstimmungen über Verfassungs- und Gesetzesvorlagen und wertete die Resultate aller Abstimmungen aus einem Zeitraum von über 30 Jahren (1981 bis 2014) Gemeinde für Gemeinde aus. Das Ergebnis: In den 1980er-Jahren war die politische Landschaft der Schweiz noch maßgeblich vom ,Röstigraben' geprägt, danach näherten sich die Städte über die Sprachregionen hinweg einander an. Das heißt: War damals die Wahrscheinlichkeit höher, dass eine Einwohnerin Zürichs oft gleich stimmte wie die eines unweit gelegenen Dorfes, so ist es heute wesentlich öfter der Fall, dass etwa die Stimmabgabe einer Zürcherin und eines Genfers übereinstimmen - und beide sich zumeist anders entscheiden als die Bewohner_innen ländlicher Gemeinden in ihrer jeweiligen Nähe.

Um die Dynamik solcher Veränderungen zu verstehen, müssen vor allem demographische Verschiebungen beachtet werden. So zeigte sich 
beispielsweise bei der österreichischen Präsidentschaftswahl nicht so sehr ein reiner Stadt-Land-, sondern vielmehr ein Wachstums-SchrumpfungsGegensatz: „Bei der Wahl siegte der Rechtspopulist Norbert Hofer von der FPÖ dort, wo Österreich schrumpft und die Männer in der Überzahl sind. Der Grüne Alexander Van der Bellen gewann, wo Österreich wächst und die Frauen hinziehen." (Gasser et al. 2016: 19)

In Deutschland wird die demographische Divergenz seit einigen Jahren zunehmend unter dem vom Ökonomen Harald Simons geprägten Schlagwort ,Schwarmstädte، diskutiert (vgl. Simons/Weiden 2015). Dieser Ansatz betrachtet Zusammenhänge zwischen Binnenmigration und ,natürlichen` Bevölkerungsveränderungen, das heißt der Veränderung von Geburtenraten. Durch den sogenannten ,Pillenknick' sind die Jahrgänge ab Mitte der 1970erJahre „deutlich dünner besetzt“, so Simons (zit. nach Eisenring 2016). Da die heute etwa Vierzigjährigen und die Angehörigen der ihnen nachfolgenden Kohorten „weniger geworden seien, ziehe es sie dorthin, wo sie ihresgleichen träfen“ (ebd.).

Dies lässt sich durch Untersuchungen des Kohortenwachstums nachweisen, der Veränderung der Anzahl Angehöriger bestimmter Geburtsjahrgänge in Gebietseinheiten. In der ursprünglichen Schwarmstädte-Studie stand die „Ausbildungs-“ und „Berufsanfängerwanderung“ der 20- bis 34-Jährigen (Jahrgänge 1979-1993) von 2008 bis 2013 im Zentrum (Simons/Weiden 2015: 12ff.). Hier stechen aus den Landkreisen und kreisfreien Städten Deutschlands 30 Schwarmstädte deutlich hervor. Darunter sind die meisten der größten Großstädte, aber auch etliche kleinere und gemeinhin als unauffälliger wahrgenommene Universitätsstädte wie Erfurt oder Darmstadt (Simons/Weiden 2015: 28).

Simons' eigene politische Folgerungen aus diesen Befunden mögen fragwürdig erscheinen: Er plädiert beispielsweise dafür, Wohnungsnot in Großstädten möglichst gar nicht zu bekämpfen, denn wenn „der Bund hilft, Berlin billiger zu machen“, dann sei dies „Umverteilung auf Kosten anderer Regionen "(zit. nach Tutt 2017). Nichtsdestotrotz verweist der SchwarmstadtDiskurs auf einige zweifellos relevante Aspekte der heutigen Stadt-LandDynamik. Sowohl wissensbasierte Ausbildungswege und Berufspraktiken als auch ein funktionierender mating market scheinen auf eine gewisse urbane Dichte angewiesen zu sein. Die bereits erwähnte Tatsache, dass die Landflucht vielerorts seit Jahrzehnten überwiegend weiblich geprägt ist, führt zu einem Fertilitäts-Migrations-Gefüge, durch das die Schwarmstädte ihr Anderes demographisch stets mitproduzieren: Ihr Wachstum korreliert mit der Abwanderung insbesondere der „sozialen Aufsteiger“ aus schrumpfenden Regionen, „die dadurch nach Art einer Abstiegsspirale weiter an Anziehungskraft und Lebensqualität verlieren und gesellschaftlich unsichtbar werden“ (Reckwitz 2017: 392).

\section{Ländliche Verzweiflung}

Die Befunde von Journalist_innen und politischen Kommentator_innen, die versuchen, die Präferenz für rechte Ideen in ,abgehängten Gebieten` zu ergründen, fallen nicht immer ausreichend differenziert aus, wie Claus Leggewie treffend kommentiert hat: 
„Aus dieser Vogelperspektive gibt es ein klares Oben (die Eliten) und ein klares Unten (das Volk), das sich auch räumlich niederschlägt: reiche Boomtowns versus armes Hinterland [...]. Abgehängte stehen, um im Bild zu bleiben, herum wie Waggons, die an einer verlassenen Bahnstation von einem Schnellzug abgekoppelt und vor Jahren dort stehen gelassen wurden. Das sei das Lebensgefühl vieler Menschen ,draußen im Lande', lautet die populistische Opfererzählung.“ (Leggewie 2017: 2)

Doch bei aller Skepsis gegenüber einem dualistischen Narrativ: Abwanderung und infrastrukturelle sowie ökonomische Nachteile sind vielerorts real, die Wahrnehmung, „dass die Zukunft woanders stattfindet“ ist wirkmächtig: „Die Jungen, die Frauen, die gut Ausgebildeten, sind ohnehin schon weg. Das Lebensgefühl der Peripherie ist das des Verlusts.“ (Rada 2017: 11)

Dabei erscheinen die Verhältnisse im deutschsprachigen Raum noch vergleichsweise mild. Um die potentielle politische Wucht von Stadt-LandGegensätzen nachzuvollziehen, lohnt sich deshalb der Einbezug entsprechender US-amerikanischer Debatten. Nicht, um ungleiche Raumrelationen über einen Kamm zu scheren, sondern um den Blick für diejenigen Aspekte von ,Urbanität ${ }^{\star}$ - und deren Gegenstück - zu schärfen, die gerade nicht nur räumlich funktionieren. Dabei ist eine Betrachtung des real existierenden, mehrheitlich weißen ländlichen Amerika durchaus instruktiv. Dessen Beschreibungen aus jüngerer Zeit scheinen - unabhängig von ihrer politischen Stoßrichtung - um das Leitmotiv ,despair' zu kreisen.

Eine eindrückliche ethnographische Untersuchung dieser Lebenswelten hat jüngst die Soziologin Arlie Hochschild mit Strangers in Their Own Land (2017) vorgelegt. Ihre Studie über Anhänger_innen des ultrarechten TeaParty-Flügels der Republikanischen Partei in Louisiana geht vom Paradox aus, dass deren Wahlentscheidungen mit deprimierender Zuverlässigkeit gegen ihre eigenen ökonomischen Interessen ausfallen: Louisiana liegt unter den US-Bundesstaaten auf den hintersten Rängen hinsichtlich zahlreicher Einkommens-, Bildungs- und Gesundheitsparameter und erhält über 40 Prozent seines Etats aus Washington DC. Trotzdem sind die TeaParty-Dogmen gegen jegliche staatliche,Einmischung in ,freie Märkte‘ auch unter Hochschilds eher einkommensschwachen Gesprächspartner_innen weit verbreitet. Daran ändert auch die verheerende Umweltverschmutzung nichts, die mit der Erdölgewinnung an der Küste des Golfs von Mexiko einhergeht und unmittelbar an „das biologische Eigeninteresse an Gesundheit und Leben" rührt (ebd.: 25).

Hochschild referiert die sozialwissenschaftlichen Debatten über den Rechtsdrall eines Großteils der amerikanischen Bevölkerung während der vergangenen Jahrzehnte kritisch (u. a. Frank 2004). Ihr eigener Erklärungsansatz basiert auf einer, wie sie es nennt, ,deep story', mittels derer sie die Ansichten und Gefühlswelten ,hinter ' den konkreten Äußerungen ihrer Informant_innen auf den Punkt zu bringen versucht. In dieser Erzählung stehen die Tea-Party-Wähler - typischerweise männlich und weiß - in einer langen Schlange von Menschen, die zum American Dream führt, der gewissermaßen als die biblische,City upon a Hill' gedacht wird. Sie stehen irgendwo in der Mitte, hinter ihnen vermehrt Schwarze, Latin@s, Frauen. Die Warteschlange 
bewegt sich immer langsamer, scheint schließlich stehen zu bleiben. Die in der Mitte Wartenden gewinnen den Eindruck, dass andere sich an ihnen vorbeidrängen, mit Unterstützung von Affirmative-action-Programmen und anderen staatlichen Maßnahmen. Sie scheinen die Anerkennung zu erhalten, die den von Hochschild befragten Tea-Party-Wählern zunehmend versagt bleibt. Die Folgerung der Autorin ist, dass bei deren Wahlentscheidungen ein ,emotionales Eigeninteresse über ein ökonomisches triumphiert; sie machen aus ihrem Kummer eine Tugend.

\section{Die ,kulturell Abgehängten` als Reaktionäre}

Eine solche, mit früheren Untersuchungen zur ,autoritären Persönlichkeit (vgl. Benicke 2016) verwandte Analyse kommt rechten Gedanken- und eben Gefühlswelten auch deswegen auf die Spur, weil sie zunächst nicht nach deren Berechtigung fragt. Das Bild vom Stehen in der Warteschlange ist auch bereits auf seine Übertragbarkeit auf den deutschen Kontext abgeklopft worden (Dörre 2018). Tatsächlich ist der Kern von Hochschilds ,deep story “ dass „Weiß-Heterosexuell-Mann-Ländlich-Religiös-Sein (wieder) Geltung erlangen“ soll (Hauser 2017) - auch in den Stimmungslagen hiesiger Rechts-Wähler erkennbar (und denjenigen von Wählerinnen, zumal es nicht isoliert um ,Männlichkeit' geht, sondern um eine konventionelle Geschlechterordnung). In Deutschland lenkte gerade der Erfolg der AfD bei der Bundestagswahl 2017 die Aufmerksamkeit auf Bevölkerungsgruppen, die sich eben weder ökonomisch noch notwendigerweise räumlich, sondern „kulturell abgehängt“ fühlen (Lengfeld 2017b): Diejenigen, „die die Modernisierung der Bundesrepublik nicht mittragen “ (Rada 2017: 11) und sich folglich, in den Worten des AfD-Vorsitzenden Alexander Gauland, „unser Land zurückholen“ wollen. Man hat es hier durchaus mit einer im Wortsinn reaktionären Gesinnung zu tun, dem tiefsitzenden Wunsch, zahlreiche gesellschaftliche Entwicklungen rückgängig zu machen. Gaulands Co-Vorsitzender Jörg Meuthen brachte dies auf die Formel, man wolle „weg vom links-rot-grün [...] versifften Achtundsechziger-Deutschland“ (vgl. dazu Stokowski 2019).

Insofern legt dieser ,Backlash“ zumindest semantisch nahe, dass eine „Fundamentalliberalisierung" der (west-)deutschen Gesellschaft (Herbert 2002: 7 , mit Bezug auf Jürgen Habermas) in vielen Bereichen tatsächlich stattgefunden hat: Die Pluralisierung von Geschlechterrollen und Familienbildern, die schrittweise rechtliche Gleichstellung homosexueller Beziehungen, und, bei allem weiterhin vorhandenen institutionellen Rassismus, eine (auch medial) gesteigerte Sichtbarkeit ethnischer Vielfalt. Durch die Augen der Rechten betrachtet sei diese „real existierende Welt der Bundesrepublik“, so der taz-Redakteur Jan Feddersen (2017: 5), ein kulturelles und politisches „Volldebakel“: „Nichts von dem, wie sie sich Deutschland vorstellen, und zwar in welchen Grenzen auch immer, funktioniert so, wie sie es gern hätten. Schlimmer noch: Es wird einfach nicht besser. Jeden Kampf haben sie verloren, manchen über Nacht, andere auf längere Sicht.“ (Ebd.)

Dieses rechte Phantasma belegt im Umkehrschluss selbstverständlich nicht, dass die Bundesrepublik und andere gegenwärtige Gesellschaften tatsächlich emanzipierte Schlaraffenländer wären - darauf wird 
zurückzukommen sein. Nichtsdestotrotz basieren gegenwärtige rechte Gefühlswelten, mit Harmut Rosa (2016) gesprochen, offensichtlich stark auf Verlusten an „Resonanz“. Nicht nur ,fremd im eigenen Land" zu sein, sondern überhaupt die Welt nicht mehr zu verstehen, ist offensichtlich ein zentrales Motiv in der Gefühlslage der als ,kulturell abgehängt' Beschriebenen. Es geht ihnen oft um die Verteidigung gefühlt, eben noch' akzeptierter Einstellungen. Was soll denn ,plötzlich“ so schlimm daran sein, dunklere Hautfarben ,exotisch' zu finden oder Homosexualität einfach nur zu tolerieren? Im CoenBrüder-Film „The Big Lebowski“ tritt der so denkende Archetyp in einer streckenweise noch harmlos-anrührend erscheinenden Variante als traumatisierter Vietnamkriegsveteran Walter Sobchak auf, der anlässlich einer vermuteten Schummelei beim Bowling eine Pistole zieht und schreit: „Has the whole world gone crazy?" Es ist kein Zufall, dass die Kritik an traditionellen Geschlechterrollen von Rechten als ,Genderwahn' diffamiert wird.[4] Auch die Affinität zu Verschwörungstheorien passt dazu: Die Welt kann sich doch gar nicht in diese ,irre' Richtung bewegen, ohne dass dahinter ein perfider Plan steckt. Daraus wiederum erwachsen eine meist höchstens einen Schritt von vulgären bis genozidalen Gewaltphantasien entfernte NotstandsRhetorik sowie völlig übersteigerte Zeitdiagnosen. So fürchtet sich der PiSPolitiker und ehemalige polnische Außenminister Witold Waszczykowski nicht nur vor einem „neuen Mix von Kulturen und Rassen“, sondern auch vor einer „Welt aus Radfahrern und Vegetariern“.[5] Die Gefährdung des Heiligsten scheint im Trivialsten auf; jeder Veggieburger wird zum Signum eines epochalen Niedergangs.

\section{6. ,Kosmopolitismus' versus ,Kommunitarismus'? Urbanität im Wertekonflikt}

Indizien für diese von Rechten beklagte, Verkommenheit' finden sich gehäuft in Großstädten, wobei es den antiurbanen rechten Vorstellungswelten auf die tatsächlichen dortigen Verhältnisse nicht ankommt. ,Neukölln“ ist für die AfD-Propaganda eine Chiffre. Wie das Zusammenleben von Menschen unterschiedlichster soziokultureller Herkunft gelingt und wo es Probleme gibt, welche Fundamentalismen wirklich gefährlich und welche Solidaritäten wirklich hilfreich sind, wie Stadtentwicklung auch und vor allem ökonomisch funktioniert - dieser Komplexität hat sich noch kein rechtes Pamphlet gestellt. Womöglich auch, weil die realen urbanen Multi-Herkunfts-Gesellschaften des 21. Jahrhunderts das zentrale Begründungsproblem aller rechten Gedankengebäude bloßstellen, die im Grunde nach wie vor auf der Idee von Geburtsvorrechten beruhen und damit nicht erst seit einigen Jahrzehnten ein Legitimationsdefizit haben, sondern seit der Französischen Revolution. Von außen gesehen dienen Selbstwahrnehmungen wie die von Hochschild herausgearbeitete ,deep story“ in erster Linie der „Verteidigung von als gerechtfertigt erachteten und durch den Aufstieg von Außenseitern als gefährdet wahrgenommenen ökonomischen, sozialen und kulturellen Vorrechten“ (Lessenisch 2018: 9).[6]

Als dreisteste Herausforderung überkommener „Etabliertenvorrechte“ (vgl. Groß/Zick/Krause 2012) empfinden heutige Rechte offensichtlich Migrationsbewegungen, die ebenjene „Sackgasse“ erhellen, in der „rechtes 
Denken“ endet, das die Idee des „eigenen Volkes“ als „transzendentale, also vorempirische Bedingung" setzt (Nassehi 2015: 296). Die Einsicht, dass eine ,Geburtslotterie‘ als Begründung für generelle Einschränkungen der Bewegungsfreiheit mit der Idee von frei und gleich an Würde und Rechten geborenen Menschen konzeptionell genauso unvereinbar ist wie Ungleichbehandlungen aufgrund von ,Stand', ,Rasse', Geschlecht oder sexueller Orientierung (vgl. Cassee 2016), scheint oft zu einer kognitiven Dissonanz zu führen, die durch eine regressive Radikalisierung aufgelöst wird: Anstatt die Sackgasse zu verlassen macht man sich lieber ein Weltbild zu eigen, das gleich alle Dimensionen menschenrechtlicher Gleichheit verwirft und mehr oder weniger unverhohlen patriarchal-autochthone Vorrechte befürwortet.

Der Ort, an dem die Bevorzugung jener, die ,schon immer hier gelebt haben', am stärksten in Frage gestellt wird, an dem die Überwindung von Geburtsvorrechten zumindest denkbar scheint, ist die „Arrival City“ (Saunders 2011), die stets durch Zuwanderung geprägte Großstadt - wobei im globalen Maßstab nachrangig erscheint, ob es sich dabei um transnationale oder Binnenmigration handelt. „That place where we really wanna go“ liegt vielleicht nur ein paar Highway-Meilen entfernt, doch für die von Springsteen besungenen „tramps like us“ ist er ein neuer Kontinent. Sich aufmachen, Wagnisse eingehen, oft genug unfreiwillig, und dabei doch immer auch mit der Chance verbunden, neue Welten zu entdecken - gerade dieser affektive Gehalt von Mobilität und Urbanität erscheint Rechtswähler_innen nach wie vor als etwas ihren eigenen Werthaltungen fundamental Entgegenstehendes. So waren den für eine Studie von zwei Marktforschungsinstituten befragten AfD-Wähler_innen diejenigen Werte wichtig, die schon in der WertewandelForschung der 1970er-Jahre als „traditionell“ galten: Zuverlässigkeit, Treue, Respekt. Unerwähnt blieben in ihren Äußerungen demgegenüber nicht nur „individualistische Werte“ wie Selbstverwirklichung oder Erfolg, sondern auch „kreative Werte“ wie Fantasie oder Neugierde und „soziale Werte“ wie Toleranz oder Empathie (Wieseke 2017).

Letzteres sollte man im Blick behalten, wenn man sich jenen bereits erwähnten, derzeit beliebten Deutungsmustern zuwendet, die einen binären Gegensatz „zwischen einer urbanen, informierten, sich globalisierenden, sich angleichenden Kultur und einer ländlichen, sich dagegen abschottenden Kultur" (Bozic 2016: 3) postulieren - wobei Ersterer in ökonomischer Hinsicht zumeist eher eine, (neo-)liberale a als eine soziale Ausrichtung zugeschrieben wird. Mit der Betonung einer Konfliktachse zwischen „universellen oder kosmopolitischen“ auf der einen und „partikularistischen oder kommunitaristischen" Einstellungen auf der anderen Seite (Schäfer 2017: 6) wird folglich nicht nur streckenweise eine vom antisemitischen Stereotyp des ,vaterlandslosen Kosmopoliten' geprägte Begriffsgeschichte unreflektiert fortgeschrieben (vgl. Schulz 2019). Auch geht damit zumeist die Annahme einher, dass dieser dichotome Werte- oder Kulturalisierungs-Gegensatz andere Kontroversen ersetzt beziehungsweise überlagert, insbesondere den ,alten' Links-Rechts-Gegensatz (stark vereinfacht: Sozialstaat versus ,freier ${ }^{6}$ Markt).[7] Gerade angesichts der Funktionsweisen städtischer Immobilienökonomie ist diese Folgerung jedoch, wie weiter unten gezeigt werden soll, höchst fragwürdig. 


\section{Die sortierte Wählerschaft: Kleinräumige Differenzierungen}

Zunächst stellt sich jedoch die Frage, wie man der realen politischen Diversität von Städten und Regionen analytisch gerecht werden kann, wenn der postulierte neue Hauptkonflikt wiederum mit Stadt-Land-Gegensätzen zusammengedacht wird. Denn tatsächlich spiegeln ,modernisierungsskeptische“ und ,-befürwortende“ Einstellungen „weniger ein großflächiges Zentrum-Peripherie- oder Stadt-Land-Gefälle“" (Leggewie 2017: 3) wider, sondern sind vielmehr je „in beiden Zonen vorhanden, also mikropolitisch in den Metropolen ebenso [...] wie im Hinterland“ (ebd.); und die sozioökonomische Ungleichheit, „deren Indikatoren in der unscharfen Aggregatgröße von Regierungsbezirken ermittelt werden, ist sehr viel kleinteiliger, oft liegen Prosperität und Depression nur wenige hundert Meter auseinander - auch und gerade in Städten, deren Durchschnittseinkommen [...] erst einmal keinen Grund zur Besorgnis signalisieren“(ebd.).,Strukturschwache“ Gebiete sind also keineswegs immer ländlich und umgekehrt (vgl. Hillje 2018). So gibt es etwa in Niedersachsen prosperierende ländliche Gebiete und in NordrheinWestfalen erzielt die AfD in manchen Vierteln der Ruhrgebietsstädte bessere Ergebnisse als in vielen dörflichen Gemeinden.

Auch hinsichtlich dieses Aspekts erscheint ein Blick in die USA hilfreich. Wie im Zusammenhang mit Hochschilds Studie bereits erwähnt, werden dort die Zusammenhänge zwischen (binären) politischen Gegensätzen und Geographie traditionellerweise auf Ebene der Bundesstaaten betrachtet: ,Red States 'versus ,Blue States‘. Aufgrund von Föderalismus und Zweiparteiensystem liegt eine solche Sichtweise nahe. Doch wie in der Schweiz (vgl. oben) zeigt sich auch in den USA, dass zunehmend eine kleinräumigere Differenzierung relevanter wird. Der Journalist Bill Bishop und der Soziologe Robert Cushing haben in ihrem Buch „The Big Sort“ (2008) darauf hingewiesen, dass dieAmerikaner_innen in politisch immer homogener werdenden Wohnlagen leben: 1976 waren knapp 26 Prozent von ihnen in Bezirken zuhause, die überdeutlich von einem Präsidentschaftskandidaten gewonnen wurden (landslide counties'), dieser Wert stieg bis 1992 auf 38 Prozent und lag 2004 schon bei beinahe 5o Prozent (ebd.: 1of.). Populäre (Selbst-) Diagnosen der gespaltenen amerikanischen Nation gibt es mittlerweile viele; oftmals mit historischen Herleitungen. Colin Woodard (2011) geht dabei etwa von nicht weniger als elf verschiedenen amerikanischen ,Nationen' aus. Hervorzuheben ist dabei, dass er betont, seine Beobachtungen bezögen sich auf die regional jeweils ,dominante Kultur', nicht auf die individuellen Bewohner_innen. Auch in den jeweils, rotesten' und ,blausten' counties entschließen sich schließlich zwanzig bis vierzig Prozent der Wähler_innen für die ,falsche' Partei.

In einen anderen geographischen Kontext übersetzt: Auch in LeipzigConnewitz wohnen AfD-Wähler_innen und auch im Erzgebirge gibt es Linke.

\section{Zum Beispiel Sachsen}

Die Zusammenhänge, denen dieser Text auf die Spur zu kommen versucht, lassen sich für Deutschland gut anhand von Sachsen beschreiben, dem Bundesland, das seit einigen Jahren aufs Engste mit einem starken 
Zuspruch zu rechten Ideen verknüpft ist: Die zwischen Latenz und manifester Militanz changierenden örtlichen Neonaziszenen waren der Nährboden des NSU, seit Ende 2014 veranstaltet die „Pegida“-Bewegung ihre wöchentlichen Volks-Feste der ,besorgten“ bis offen faschistischen Gefühlslagen, und zuletzt gemahnten die Aufmärsche in Chemnitz an die Hochphasen rechter Mobilisierung in den 1990er-Jahren. Auch jenseits solcher Verdichtungsmomente bricht sich in Sachsen ein aggressiver Rassismus immer wieder Bahn. Im Vergleich mit anderen Bundesländern werden hier im Verhältnis zur Einwohnerzahl besonders viele Gewalttaten gegen Geflüchtete und Anschläge auf Unterkünfte für Asylsuchende begangen (Pro Asyl 2017). Die seit der deutschen Wiedervereinigung von der sächsischen CDU im Alleingang oder mit deutlich kleineren Koalitionspartnern gutsherrenartig geführte Landesregierung trägt für dieses Klima zweifelsfrei eine Mitverantwortung. Immer wieder wird seitens verschiedener Behörden das Problem gewaltbereiter rechter Strukturen verharmlost; zugleich gehen die sächsische Polizei und Justiz gegen antifaschistischen Protest oft mit äußerster Härte vor. Offiziell hängt man einer gleichmacherischen Extremismustheorie an (Kleffner/Meisner 2017).

Dieses Staatsversagen hat die Sozialisierung von mittlerweile mehreren Generationen eines rechten Milieus mit ermöglicht, so dass es in Sachsen Landstriche und Kleinstädte gibt, in denen von einer rechten Hegemonie in der Alltags- inklusive der Jugendkultur ausgegangen werden muss. Diese weisen oft demographische und ökonomische Merkmale von im engeren Sinne ,abgehängten Gebieten` auf: Die Bevölkerung schrumpft und altert kontinuierlich, Bahnlinien wurden stillgelegt, Geschäfte und Schulen geschlossen und Pfarrstellen abgebaut. Die Einwohner_innen des Erzgebirgskreises erzielen die bundesweit niedrigsten durchschnittlichen Bruttoeinkommen (Brandau 2017). Doch auch in vergleichsweise wohlhabenden Regionen des Bundeslandes finden Pegida und die AfD breiten Zuspruch, insbesondere in Dresden und dessen Vororten.

In Sachsen liegt aber auch Leipzig, eine ,Schwarmstadt' par excellence, die während der letzten Jahre wie keine andere in Deutschland als Hort ,hipper' Urbanität, von Subkultur und alternativen Lebensformen medial gefeiert wurde (vgl. Volk 2013, dazu auch Eckardt/Seyfarth/Werner 2015). Exponent_innen des offiziellen Leipzig rühmen gerne die ,bürgerschaftliche' Tradition der Stadt, auch im expliziten Gegensatz zur Residenzstadt Dresden. Tatsächlich sah sich der Leipziger Pegida-Ableger „Legida“ von Anfang an mit zahlenmäßig überlegenem Gegenprotest konfrontiert, dem sich zeitweise auch der Oberbürgermeister und zahlreiche Stadträt_innen anschlossen (notabene aber kaum je CDU-Vertreter_innen), während aus dem Dresdner Establishment immer wieder Zustimmung zu den rechten Aufmärschen zu vernehmen ist und die dortigen Gegendemonstrant_innen fast immer in der Minderheit blieben. Legida dagegen stießjenseits eines hart rechten Kernmilieus kaum auf Anklang. Das mag auch daran liegen, dass mit der ,Schwarm'-Zuwanderung der letzten Jahre in Leipzig vor allem eher linke Milieus gewachsen sein dürften, Grünen-affine Mittelschichtsfamilien ebenso wie die studentisch geprägte bewegungslinke Szene.

Dies sollte nicht zum Fehlschluss verleiten, Leipzig sei insgesamt eine Insel der Weltläufigkeit im ,dunkeldeutschen' Sachsen. Der Alltagsrassismus kann 
in der Großstadt insofern noch perfider sein als dass ihn Nichtbetroffene weniger wahrnehmen als dort, wo er offener zu Tage tritt. Richtet man den Blick wiederum auf binnenräumliche Differenzierungen und in Wahlresultaten gemessene politische Einstellungen, so lässt sich konstatieren, dass nur einige der relativ zentral gelegenen Gründerzeitviertel Leipzigs der Vorstellung von ,progressiver Urbanität‘ mit einer liberalen bis linken Mehrheit entsprechen.[8] Dies ist allerdings vielerorts nicht anders. Auch in Berlin oder im Ruhrgebiet ergeben sich in weniger dichten und zentrumsnahen Gebieten - seien es Großsiedlungen oder Einfamilienhausquartiere - deutlich ,modernisierungsskeptischere‘ Mehrheiten.

Um der konkreten Konstituierung dieser politischen Verräumlichung auf die Spur zu kommen, ist wiederum ein Blick auf demographische Dynamiken nötig, das heißt hinsichtlich des Fallbeispiels Sachsen auf die Binnenmigration, die hier besonders deutlich einem ,Schwarmverhalten' entspricht (Simons/ Weiden 2016): Während der letzten Jahre sind fast nur die Großstädte in relevantem Ausmaß gewachsen, und innerhalb dieser am stärksten ebenjene urban-dicht-progressiven Stadtteile. Daneben ergeben sich durch eine in Leipzig und Dresden mittlerweile manifeste Wohnungsknappheit erste Suburbanisierungs- beziehungsweise „Überschwappeffekte“ (Münter/ Osterhage 2018: 14ff.) - von den politischen Vertreter_innen des ländlichen Raumes gerne absichtsvoll zweckoptimistisch mit einer ,neuen Landlust ${ }^{\text {‘ }}$ verwechselt.

Die vielen Einzelentscheidungen, die hinter diesen Wanderungszahlen stehen, sind somit offensichtlich nicht selten auch Entscheidungen gegen ein Leben an Orten, die politisch stark rechts geprägt sind, und für eines an den landesweit ,linksten' Orten. Das heißt nicht, dass jede_r, die_der aus einem erzgebirgischen Dorf in einen Leipziger Gründerzeitstadtteil zieht, dies aufgrund ihrer_seiner politischen Einstellung tut. Doch zum einen sind nicht wenige Großstädter_innen den rechten Hegemonien in den Dörfern ihrer Kindheit entflohen (vgl. autobiographische Berichte wie jüngst den von Daniel Schulz 2018) und zum anderen muss man nicht auf bestimmte Umzugsgründe fokussieren, um sich darüber zu wundern, dass die Binnenmigrant_innen als solche in den aktuellen Debatten kaum vorkommen. Dabei können gerade sie den Blick darauf lenken, dass eine statische Dichotomie von städtischen versus ländlichen Identitäten den realen Biographien unzähliger Individuen nicht gerecht wird: Man ,ist‘ nicht, einfach so', wenn man,von dort' ist - doch zu oft bleibt jenen, die eine ressentimentgeprägte Vorstellung von Gemeinschaft nicht mittragen wollen, nur das Abhauen von dort.

\section{Eine Welt aus Stadtstaaten?}

Das bisher Gesagte ließe sich salopp wie folgt zusammenfassen: Rechte politische Einstellungen sind am dominantesten in ländlichen Gebieten, deren Bevölkerung schrumpft. Als linke_r Städter_in könnte man sich folglich zu demographischem Optimismus verleiten lassen. Stirbt das Ressentiment einfach aus?

Eine solche Perspektive nahm in gewisser Weise der US-Politikwissenschaftler Benjamin Barber ein. In einem seiner letzten Texte schrieb der 
im April 2017 Verstorbene, Donald Trump versuche, gegen die „winds of change“ zu segeln: „Global demographics are against him, as are American demographics; the reality of urbanization is against him; the mobility of peoples is against him. " (Barber 2016) Tatsächlich besteht, wie gesagt, die ,neue' Rechte aus weitestgehend reaktiven Bewegungen, sind Worte wie ,Backlash' durchaus akkurat: Weil Gesellschaften vielfältiger werden und zumindest potentiell mehr und verschiedenartigeren Menschen Mitsprache ermöglichen, kommt es zu Abwehrreaktionen eines weiß-männlich-heterosexuellen „Normalitarismus“ (Schaible 2018), die gerade in ihren extremsten Formen, wie jüngst beim Attentat von Christchurch, selber in verquerer Weise auf demographische Prozesse Bezug nehmen. Eine Position, die Geburtsvorrechte befürwortet, fürchtet die mit dem selbstermächtigenden Potential von Migration einhergehenden Umwälzungen in gewisser Weise zurecht. Allerdings legt das hier Erörterte nahe, dass die transnationalen Verschiebungen, auf die sich die Obsessionen der Rechten fast ausschließlich richten, deren gesellschaftlichen Idealvorstellungen womöglich weniger rasch und unmittelbar abträglich sind als die sich vielerorts in (Re-)Urbanisierung übersetzenden Bevölkerungsveränderungen innerhalb von Staaten.

Mit deren Dynamik haben freilich die Modi der politischen Repräsentation nicht Schritt gehalten. Wahlsysteme gehen überwiegend von in mehr oder weniger großflächigen Einheiten räumlich zusammengefassten Gruppen aus, denen durch gemeinsame Vertretung implizit eine gewisse politische Homogenität unterstellt wird. Die so in parlamentarischen Systemen verankerte Vorstellung einer primär regional definierten Wählerschaft steht in klarem Widerspruch zur oben analysierten heutigen Verräumlichung politischer Einstellungen, die durch einen hohen Grad an Übereinstimmung zum einen innerhalb von kleinräumigen Gebieten, zum anderen über große Distanzen hinweg geprägt ist. (,Linke(re) ${ }^{`}$ Stadtteile in verschiedenen Städten ähneln einander wie erwähnt viel stärker als ihrem jeweiligen Umland.)

Rückständige föderalistische Repräsentationsmodi gehen oft auf Staatsgründungsprozesse zurück, in denen der Ausgleich zwischen regionalen Gegensätzen ein wichtiges Anliegen war. So billigte die Schweizer Bundesverfassung von 1848 den im vorangegangenen Sonderbundskrieg unterlegenen katholischen Kantonen auf Bundesebene einen überproportionalen Einfluss zu: Im Ständerat, der gleichberechtigten zweiten Parlamentskammer, ist jeder Kanton unabhängig von der Bevölkerungszahl mit zwei Sitzen vertreten. Dieses im US-Senat schon ein halbes Jahrhundert früher zur gleichen Form gelangte Wahlsystem unterminiert das Prinzip von ,eine Person, eine Stimme noch krasser als das Elektorenstimmenprinzip bei der Präsidentschaftswahl, dem die Welt die Siege der - man kann nicht oft genug daran erinnern - in absoluten Stimmen unterlegenen Kandidaten George W. Bush und Donald Trump verdankt. Beide Mechanismen gehen im amerikanischen Kontext letztlich auch auf Kompromisse zurück, die in der Entstehungszeit der Verfassung von den sklavenhaltenden Südstaaten durchgesetzt wurden. Durch antiquierte Regionalkammern sind die wahlberechtigten Einwohner_innen kleinerer Gliedstaaten gegenüber denjenigen größerer auf Bundesebene massiv überrepräsentiert; in der Schweiz gemäß aktuellen Bevölkerungsdaten bis zu circa 41-, in den USA bis zu etwa 67-fach. In beiden Ländern geht dieses Ungleichgewicht zulasten der am stärksten 
urbanisierten Gebiete. (Dies gilt auch für das Gerrymandering, die unverblümt parteiische Grenzziehung von Wahlkreisen.)

Auch abgesehen von solchen numerischen Schieflagen fehlt den großen Städten in auf Flächenländern basierenden föderalen Systemen ein politisches Gewicht, das ihrer wirtschaftlichen und kulturellen Bedeutung gerecht würde. So gilt für die USA: „Power may be decentralized [...], but it devolves to the state, not the city" (Graham 2017: 24), und die Staaten scheuen sich, wenn sie in republikanischer Hand sind, nicht, die Eigenständigkeit demokratisch dominierter Metropolen massiv zu beschneiden. In Deutschland lässt sich Ähnliches beobachten, wenn man sich etwa wiederum die reichhaltigen Spannungsverhältnisse zwischen der Stadt Leipzig und der sächsischen Landesregierung vergegenwärtigt.

Hier zeigt sich eine fundamentale Diskrepanz: Einerseits basieren Ökonomie sowie Ideen- und Bildwelten der globalisierten Gegenwart maßgeblich auf einem (hierarchischen) Netzwerk von Städten (Sassen 1991); andererseits wird die - tendenziell jüngere, besser gebildete und ethnokulturell vielfältigere - constituency der Städter_innen im nationalstaatlichen Rahmen politisch oft klein gehalten. In amerikanischen Debatten wird angesichts dessen auf die Notwendigkeit verwiesen, politisch-räumliche Bezugsgrößen neu zu denken. Als Baustein für eine den Herausforderungen des 21. Jahrhunderts gewachsene, notwendigerweise globale polity taugt der Nationalstaat offensichtlich kaum. Wie wäre es also stattdessen mit einer Welt aus Stadtstaaten? „If Mayors Ruled the World“ - so überschrieb der bereits erwähnte Benjamin Barber (2013) diesen Denkhorizont. Er verwies auf die Mannigfaltigkeit bereits existierender Zusammenschlüsse zwischen Stadtregierungen, die beispielsweise in Umweltfragen handlungsfähiger erscheinen als Nationalstaaten.

Dabei weist der Urbanitätsbegriff des Demokratietheoretikers Barber über den vereinfachenden Gegensatz zwischen ,Kosmopolitismus' oder ,Universalismus' einerseits und ,Kommunitarismus“ oder ,Partikularismus andererseits hinaus. Diesen hatte Barber selbst bereits kurz nach Ende des Kalten Krieges nicht nur thematisiert, sondern auch - deutlicher als ihm nachfolgende Autor_innen - problematisiert: Die beiden „axial principles of our age“, nämlich „tribalism“ und „globalism“, würden, so Barber, einander in jeder Hinsicht entgegenstehen, außer in einer: „They may both be threatening to democracy.“ (Barber 1992: 53) Das Gegenmittel zu den destruktiven Kräften religiöser und nationalistischer Fundamentalismen einerseits und eines unregulierten Kapitalismus andererseits („Jihad vs. McWorld“) sah Barber in einer funktionierenden politischen Repräsentation auf lokaler sprich städtischer - Ebene. Kurz vor seinem Tod begründete er dies unter aktuellen Vorzeichen vor allem damit, dass Städte Nationalstaaten als universalistische Institutionen den Rang abgelaufen hätten: „Cities speak to global common goods - marriage rights, minimum wage, climate action, creative culture, refuge for immigrants - while nations have grown parochial and xenophobic.“ (Barber 2016).

An der Neuheit dieses Befundes mag man, was seine Dringlichkeit keineswegs mindert, Zweifel anmelden: Das schmutzige Geheimnis des Nationalstaats ist seit jeher, dass er, wie der Name sagt, den Staat nicht ohne die Nation bietet, die res publica nicht ohne Abstammungsideologien, 
Bürgerrechte nicht ohne Ausschlüsse. Entsprechend trieb die Konstitution von nicht-nationalstaatlichen Vermittlungsinstanzen zwischen Individuum und Gesellschaft schon die im Angesicht einer sich entfaltenden urbanen Moderne schreibenden Klassiker der Soziologie um (vgl. dazu Hawkins 1994, Isin 2002: 231ff., Haupt 2004). Eine ,Beheimatung' von Menschen, die typischerweise in urbanen Kontexten - Aufgehobenheit ermöglicht, ohne zur antiindividualistischen dörflichen Zwangsgemeinschaft zu regredieren, ist das bleibende „Bezugsproblem“ der Moderne (Nassehi 2016: 76).

Ein Modell (quasi-)souveräner Städte als Vehikel für eine andere, humanere Verschränkung von Solidaritätund Differenz zu betrachten, erscheint tatsächlich reizvoll - und höchst aktuell: Gerade im Kontext von Migrationsthemen haben sich in den letzten Jahren weltweit städtische Bewegungen gebildet, die für Konzepte eines von Nationalstaatlichkeit unabhängigen städtischen Bürgerrechts (urban citizenship; vgl. Hess/Lebuhn 2014) kämpfen und, insbesondere in den USA, für die Verweigerung der Zusammenarbeit mit den bundesstaatlichen Abschiebebehörden (sanctuary cities; vgl. Scherr/ Hofmann 2016). In Spanien sind - auch aus wohnungspolitischen Protesten heraus - kommunale Wahlbündnisse entstanden, die sich explizit als ,munizipalistisch'verstehen (vgl. Zelik/Bruchmann/Candeias 2016). Diese haben beachtliche Erfolge errungen, darunter die Wahl der Anti-ZwangsräumungsAktivistin Ada Colau zur Bürgermeisterin von Barcelona. Hier lassen sich historische Verbindungslinien zum Munizipalsozialismus ziehen, den sozialdemokratische Stadtregierungen des frühen 20. Jahrhunderts propagierten, doch scheinen sich heutige Munizipalismen nicht nur eine Verbesserung des Lebens in den Städten auf die Fahnen zu schreiben, sondern auch das Städtische an sich zu zelebrieren - und womöglich zu idealisieren. In den USA ist diesbezüglich eine spezifische Tradition erkennbar, die mindestens bis zu Jane Jacobs' The Death and Life of Great American Cities (1961) zurückreicht und in jüngerer Zeit verschiedene, bisweilen durchaus technokratisch-kapitalistisch gestimmte Lobgesänge auf die Stadt als Motor modernen Lebens hervorgebracht hat (vgl. bspw. Glaeser 2011).

\section{Knotenpunkte statt Leuchttürme}

Das Grundproblem solcher produktivitätseuphorischer Urbanitätsdiskurse ist, dass ihr Begriffdes Städtischen die Dichotomie zwischen,Kosmopolitismus und ,Kommunitarismus unkritisch fortschreibt. Dabei ist Prosperität weder per se progressiv noch notwendigerweise urban. Dies zeigt etwa eine Analyse der Stimmenverteilung in counties mit unterschiedlichen durchschnittlichen Einkommen, Bildungsgraden und nichtweißen Bevölkerungsanteilen bei der letzten US-Präsidentschaftswahl (Silver 2016). Auch diskursiv ist die Abwertung der ,Unproduktiven', die eine ökonomistische Sicht auf ,kosmopolitische‘ Urbanität mit sich bringt, alles andere als emanzipatorisch. Im Gegenteil wird „Urbanität“ so „zum Codewort für Arroganz, Kälte und Zynismus“ (Leggewie 2017: 2). Doch hat die sich in den letzten Jahren weltweit verschärfende Wohnungsnot in den Städten mittlerweile auch Richard Florida, einstige Galionsfigur eines solchen am Problem sozialer Ungleichheit desinteressierten urbanen ,Kreativitäts'-Liberalismus, zur Umkehr bewegt. „I found myself confronting the dark side of the urban revival 
I had once championed and celebrated“, bekennt er in bester Paulus-Manier (Florida 2017a: xvii), predigt nun einen „inclusive urbanism“(ebd.: xx) - und fordert als Rahmen für dessen Umsetzung, Benjamin Barber folgend, die „United Cities of America“ (Florida 2017b).

Tatsächlich kann die Idee munizipaler Souveränität deutlicher ,links aufgeladen werden, wenn man sie mit dem spezifischen Potential urbaner Räume zusammendenkt, jene Orte hervorzubringen, an denen man in der Dienstleistungs- und Wissensökonomie nicht nur arbeitet, sondern sich auch vergemeinschaftet und im besten Fall politisch organisiert: „The metropolis is to the multitude what the factory was to the industrial working class." (Hardt/Negri 2009: 250) Und die arbeitende Bevölkerung in den Städten des 21. Jahrhunderts ist kulturell, ethnisch und geschlechtlich selbstverständlich wesentlich vielfältiger als es den von fordistischer Blue-collar-Folklore evozierten Bildern entsprechen würde (vgl. Dowling/van Dyk/Graefe 2017: 419).

Bezeichnenderweise sind jedoch gerade kulturelle Konfliktlinien betonende Gesellschaftsbeschreibungen gegenüber der Interdependenz von Vielfalt und Ungleichheit ziemlich blind. Wenn Andreas Reckwitz (2018: 43) Akademiker_innen pauschal zur „neuen Mittelklasse“ erklärt, die von einer prekarisierten „neuen Unterklasse“ klar abgrenzbar sei, muss er sich fragen lassen, wie sehr eine solche Diagnose durch seine persönliche Perspektive als Lehrstuhlinhaber geprägt ist. Gerade in den deutschen Großstädten - man könnte hier wiederum Leipzig als treffendes Beispiel anführen - lebt ein Großteil des bildungsaffinen und politisch progressiven Bevölkerungssegments in ökonomisch alles andere als rosigen Verhältnissen. Das Missverhältnis von Einkommen und Lebenshaltungskosten wird dabei in den letzten Jahren an allererster Stelle bei den rasant steigenden Mieten spürbar (Rink et al. 2015). Es kann deshalb nicht überraschen, dass die Wohnungsfrage für Stadtbewohner_innen zum drängendsten politischen Anliegen geworden ist. Ein egalitär gedachtes ,Recht auf Stadt' muss nicht nur gegen autoritär-exkludierende, sondern vor allem auch gegen die damit verschränkten neoliberal-kapitalistischen Semantiken und Mechanismen der Ungleichheit erkämpft werden.

Gerade mit Blick auf das Wohnungsthema zeigt sich, dass diese beiden alles andere als inkompatibel sind. Unversteuerte und anderweitig unrechtmäßig erworbene Gelder spielen im Immobiliensektor aufgrund mangelnder Regulierung - auch in Deutschland - eine gewichtige Rolle (Körner 2018). Die Branche ist ein bevorzugtes Tummelfeld von Akteuren im Überlappungsbereich von privatem Reichtum, organisierter Kriminalität und politischer Macht. Dass von den autoritären Führungsfiguren, deren Aufstieg man in den letzten Jahren beobachten konnte, fast alle auf die eine oder andere Weise in zwielichtige Grundstückdeals verwickelt sind, erscheint nur folgerichtig. Das englische Wort ,landlord' für Vermieter verdeutlicht, wie viel Feudalismus im angeblich meritokratischen System ,freier Märkte' steckt, zumal wenn auf diesen mit Grund und Boden gehandelt wird. Dass das weitgehend ererbte Vermögen Donald Trumps zu einem Großteil auf der Ausbeutung von Mieter_innen und mutmaßlich massivem Betrug an der öffentlichen Hand basiert (Barstow/Craig/Buettner 2018), ist kein Zufall. Es spricht einiges dafür, dass die ethnische Segregation, die - gerade im amerikanischen Kontext - städtische Ungleichheit oft kennzeichnet, 
auch Trumps spezifischen, „psychogeographischen“ Rassismus geprägt hat (Sandifer 2016, Übers. TB).[9]

Doch hängen die städtische Wohnungsproblematik und die Auseinandersetzung mit den Rechten auch in Bezug auf deren (stärkeren) Einfluss in peripheren Gebieten zusammen. „Das Phänomen der Landflucht aus deindustrialisierten, darunter von der Agrarindustrie verlassenen Räumen [...] verschärft die sozialen Ungleichheiten und sozialpolitischen Probleme gerade auch in Städten." (Leggewie 2017: 4) Es braucht auch deshalb weiterhin für Gering- und Normalverdienende bezahlbare Wohnungen in den Großstädten, damit Zuzug an diese wenigen, jedoch stark wachsenden Orte mit progressiven Mehrheiten (vgl. oben) möglich bleibt - gerade für jene, denen aufgrund ihrer Hautfarbe, ihrer sexuellen Orientierung oder anderweitig als abweichend rubrizierten Eigenschaften ein Leben in den Zonen rechter Hegemonie nicht zugemutet werden sollte. Umgekehrt bieten die mittlerweile auch im Umland ostdeutscher Städte beobachtbaren ,Überschwappeffekte durchaus die Chance, Nischen von Urbanität dorthin zu tragen, wo bislang Abschottung und Ressentiment dominieren.[10]

Damit rücken die Zwischenräume zwischen großstädtischen Zentren und eindeutig peripheren Gebieten ins Blickfeld. Zugespitzt gesagt: Eher als in Kreuzberg oder Connewitz einerseits und womöglich wirklich ,verlorenen' Dörfern andererseits wird sich die Auseinandersetzung zwischen progressiven und reaktionären Weltbildern in suburbanen Räumen und (großstadtnahen) Klein- und Mittelstädten entscheiden. Indizien dafür finden sich vielerorts: Die counties, in denen die US-Republikaner_innen ihre (weiße) Mehrheit mittelfristig zwangsläufig verlieren werden, bestehen oft aus suburbs multiethnischer Großstädte wie Atlanta. Auch in der Schweiz galt die ,Agglo', die Ansammlung zersiedelter Vororte namentlich im Großraum Zürich, noch vor wenigen Jahren als Hochburg der rechten SVP; bei den Kommunalwahlen kam es hier jedoch jüngst zu einem für Schweizer Verhältnisse markanten Linksruck (Hotz 2018). Gleiches zeigte sich bei der letzten Oberbürgermeisterwahl in Frankfurt an der Oder, das dabei ist, sich aus Berliner Sicht von einer Pendeldestination zu einer Wohnort-Alternative zu entwickeln (Bangel 2018).

Auch das Beispiel Sachsen kann an dieser Stelle noch einmal angeführt werden. Die politisch-kulturellen Gegensätze präsentieren sich hier bei genauer Betrachtung nicht als simpler Großstadt-Land-Graben, sondern eher als Wasserscheide zwischen tatsächlich ,abgehängten ' Gebieten und solchen, die nennenswerte infrastrukturelle und kommunikative Anbindungen an urbane Orte aufweisen. Gerade im Umland von Leipzig ist die Ausbreitung alternativer Wohn- und Kulturprojekte in Kleinstädte und dörfliche Gemeinden mit annehmbarer Verkehrsanbindung zumindest in Ansätzen zu beobachten.[11]

Was könnte die am ,Hinterland“ auf den ersten Blick dezidiert desinteressierte Idee einer Welt aus Stadtstaaten in solchen Konstellationen beitragen? Viel, wenn man als Wesenskern des Munizipalen nicht Prosperität, sondern Selbstverwaltung begreift, wenn man Städte nicht als ,Leuchttürme konzipiert, sondern als Knotenpunkte vielfältiger räumlicher Relationen, wenn man folglich „eine echte Lösung der Wohnungsfrage, die der brutalen Verdrängung der Menschen mit geringem und mittlerem Einkommen 
aus den Städten einen Riegel vorschiebt“ zusammendenkt mit einer Infrastrukturpolitik, „die Leben und Arbeiten auch auf dem flachen Land wieder attraktiver macht“ (Leggewie 2017: 5).[12] An dieser Stelle könnte auch der Kommunitarismusbegriff, dem die Reduktion auf das Gegenstück zu ,Kosmopolitismus' kaum gerecht wird, (wieder) auf links gedreht werden. Seine vorletzte Popularisierungswelle verdankte er ja nicht zuletzt den Untersuchungen Robert Putnams (1993) zur spezifisch urbanen Tradition von Zivilgesellschaftlichkeit, die sich im Englischen im Begriff ,civic 'verdichtet.

Der ländliche ,Kommunitarismus' ist dagegen oft ein ausschließender, der dabei tragischerweise noch nicht einmal den inneren Zusammenhalt stärkt. Denn die Behauptung der vergleichsweise höheren gesellschaftlichen Kohäsion auf dem Land ist heute nur mehr eine konservative Lebenslüge wie etwa aktuelle Untersuchungen zum in den Städten lebendigeren und vielfältigeren Vereinswesen belegen (Priemer/Krimmer/Labigne 2018). In Deutschland zeigt sich dies wiederum besonders im Osten, wo, wie oben geschildert, während der vergangenen Jahrzehnte gerade den sozial Engagierten der Umzug in eine Großstadt oft unumgänglich schien. Es fehlt mancherorts, so drücken es auch unpolemische Beobachter_innen aus, „eine ganze Generation“ (Rennefanz 2018).

Ein den ländlichen Raum mitdenkender Munizipalismus könnte hier durch das Einfordern echter Partizipationsmöglichkeiten gegensteuern. Eine Demokratisierung der Planungspolitik (wie sie in bundesdeutschen Städten seit den 1970er-Jahren mit wechselhaftem Erfolg vorangebracht wurde) würde bedingen, dass lokale Entscheidungsautonomien und Verfügungsrechte gestärkt werden, insbesondere was Investitionen in öffentliche Infrastruktur angeht. Verbesserte Verkehrsanbindungen und gut ausgestattete Schulen (auch oder gerade wenn sie in der übernächsten Ortschaft liegen) anstelle von Austerität und Desinvestition - das würde Abhilfe hinsichtlich der tatsächlichen Probleme schaffen, auf die auch zahlreiche Rechtswähler_innen gerne verweisen (und die dadurch, dass sie Ressentiments vorgeschoben sind, nicht weniger real werden). Eine Stärkung von genuinen Einflussmöglichkeiten auf die lokale Entwicklung könnte somit auch dazu beitragen, jenes in Ostdeutschland teilweise vorherrschende Demokratieverständnis zu überwinden, gemäß dem eine als ebenso omnipotent wie alleinverantwortlich imaginierte Obrigkeit zu liefern habe, was die Mehrheit (wenn nicht, das Volk') einfordert.

Im Anthropozän wäre es jedenfalls schiere Realitätsverweigerung, die Stadt ohne das Land, geschweige denn das Land ohne die Stadt zu denken. Ein antiurbanes Klischee besagt, dass viele Städter_innen, bei der Frage, wo ihre Nahrungsmittel herkommen, ausschließlich an den Supermarkt denken würden; dem lässt sich leicht ein Bild von Landbewohner_innen gegenüberstellen, die ohne von den Zentren erdachte und bezahlte Versorgungsnetze für Energie und Wasser auch nicht überlebensfähig wären und die tatsächlich den größeren ökologischen Fußabdruck haben. Verdichtete Bauweisen sind in jedem Fall nachhaltiger, da Boden die endlichste aller Ressourcen ist. Eine Gruppe von Schweizer Architekt_innen und Planungsexpert_innen hat ihr Land bereits 2005 als eine einzige Stadt mit großen Grünflächen dargestellt (Diener et al. 2005) - im Zeitalter einer „planetary urbanization“ (Brenner 2014) die einzig adäquate Sichtweise. 
Somit kann auch nur eine explizit internationalistische Position, die auf der Unteilbarkeit von ökologischen, ökonomischen, geschlechtlichen, ethnischen und anderen Dimensionen der Gerechtigkeit beharrt, für sich in Anspruch nehmen, zeitgemäß zu sein. Wenn der Konflikt zwischen einer neoliberal-,kosmopolitischen' und einer reaktionär-,kommunitaristischen' Position, die ökonomisch beide mehr oder weniger ,rechts' sind, andere Gegensätze zu überlagern scheint, gibt es keinen Grund, eine linke Umverteilungsagenda nur in einem migrationsskeptischen nationalen Korsett für erfolgversprechend zu halten (zumal auch der ,Kosmopolitismus der Neoliberalen, die in der Regel durchaus für Einschränkungen der Bewegungsfreiheit eintreten, realiter ein sehr beschränkter ist). Gerade ein antinationaler Munizipalismus könnte, in Ulrich Becks etwas blumigen Worten, die vermeintliche Dichotomie zwischen „Wurzeln und Flügeln“ überwinden, und so ein „partikulares Weltbürgertum“ zum „gemeinsamen zivilisatorischen Nenner weltkulturell heterogener Gesellschaften" machen (zit. nach Bozic 2016: 3).[13]

Der Kampf um bezahlbaren - im besten Fall durch öffentliche und genossenschaftliche Eigentumsformen dauerhaft dekommodifizierten Wohnraum in den Städten ist dabei als linke Schlüsselstrategie zu betrachten. In ihm fließen mindestens drei essentielle Anliegen zusammen: Eine ökologische Apokalypse zu verhindern, Räume mit progressiven Mehrheiten zu sichern und auszuweiten und den Trump'schen Grundrenten-Neofeudalismus niederzuwerfen. Dass die Wohnungswirtschaft einen „lukrativen, aber unproduktiven Teil der städtischen Wirtschaft“ darstellt, „der sich leicht besteuern lässt, weil er nicht nach Offshore verschoben werden kann“ (Kuper 2017), könnte als ermutigender Fingerzeig auf mögliche Ausgangspunkte dieser monumentalen Unterfangen gelesen werden.

Dieser Artikel wurde durch Mittel des Open-Access-Publikationsfonds der Freien Universität Berlin gefördert.

\section{Endnoten}

[1] Ich danke Roman Grabolle für zahlreiche Hinweise und inspirierende Gespräche, die maßgeblich zur Entstehung dieses Textes beigetragen haben.

[2] Hierzu ausführlicher die Einleitung zu diesem Heft, ebenso zur Terminologie - in diesem Beitrag wird schlicht von, rechten' Parteien, Ideen etc. gesprochen, um mit Qualifizierungen wie ,extremistisch` einhergehende allzu bequeme Trennschärfen u. ä. zu vermeiden.

[3] Dies gilt zunächst für Europa und Nordamerika; in globaler Perspektive ist der Befund weniger eindeutig. In der Türkei lässt sich ein ähnlicher Gegensatz beobachten (vgl. Zürn 2016); vielerorts - etwa in Indien, den Philippinen oder Brasilien - verfügen ethnonationalistische, religiös-fundamentalistische oder rechtsautoritäre Bewegungen aber auch über eine starke Basis in großstädtischen Gebieten, vgl. dazu Goebel 2016.

[4] Der sich oft gewaltsam - bis hin zu misogynen Massenmorden - äußernde Antifeminismus ist ein konstitutives Element rechter Gedankenwelten, das hier nicht in angemessenem Umfang einbezogen werden kann.

[5] Vgl. Polen warnt vor,Welt aus Radfahrern und Vegetariern'. In: Zeit online, 03.01.2016. https://www.zeit.de/politik/ausland/2016-01/medienreform-polen-kritik-eukommission (letzter Zugriff am 10.10.2018). 
[6] Koppetsch (2017) spricht - wie Lessenich auf Deutschland bezogen - vom „Aufstand der Etablierten“.

[7] Eine interessante Variante dieser These stammt vom Kultursoziologen Andreas Reckwitz. Er konstatiert, im Vergleich zu einem schlichten „Kampf der Kulturen“, einen Konflikt „grundsätzlicherer und abstrakterer Art“ zwischen zwei konkurrierenden „Regimes der Kulturalisierung“, einem „öffnenden“ Regime, genannt „Hyperkultur“, dessen Leitsemantiken „Diversität“ und „Kosmopolitismus“ sind, und einem „schlieBenden“ Regime, dem „Kulturessenzialismus“, der weniger auf das Individuum als auf die Gemeinschaft abzielt, das „Alte“ und „Eigene“ valorisiert und so einen starken Antagonismus zwischen ingroup und outgroup konstruiert. Nicht originell, aber wichtig ist Reckwitz' Beobachtung, dass einander feindlich gesinnte Gruppen wie islamfeindliche europäische Rassisten und fundamentalistische Islamisten dabei „dem gleichen Muster“ folgen (Reckwitz 2016).

[8] Vgl. etwa Ergebnisse aus den Leipziger Stadtteilen, LVZ online, 20.11.2017. http://www. lvz.de/Thema/Specials/Bundestagswahl-in-Leipzig/Wahl-in-Leipzig/Ergebnisse-ausden-Leipziger-Stadtteilen (letzter Zugriff am 10.10.2018).

[9] Damit vergleichbar ist der städtische Revanchismus, den Ugo Rossi (2018) für Italien beschrieben hat- in expliziter Abgrenzung zu einer von den hier z. T. referierten amerikanischen Arbeiten abgeleiteten vereinfachenden Verortung reaktionärer Kräfte (ausschließlich) außerhalb der urbanen Zentren.

[10] Eine realistische linke Erzählung zu Binnenmigration und Suburbanisierung hat sich dabei gegen den liberal-konservativen Mythos einer angeblich wiederentdeckten ,Landlust" und die daraus erwachsende antiquierte Eigenheimförderung (aktuell in Gestalt des ,Baukindergeldes') zu stellen. Auch hier zeigt sich der Mangel an munizipaler Unabhängigkeit schmerzlich: Oft wird eine echte großstädtische Wohnungspolitik durch von Vertreter_ innen des ländlichen Raumes dominierte Parlamente verunmöglicht. Eigenständige Initiativen sind rot-(rot-)grün geprägten Städten - sofern deren Regierungen sich von neoliberalen Paradigmen ein Stück weit emanzipiert haben - am ehesten möglich, wenn sie, wie Berlin oder Hamburg, tatsächlich teilsouverän sind, oder es sich, wie München, leisten können, Maßnahmen durch eigene Steuereinnahmen zu finanzieren.

[11] Zu alternativem Wohnen in Leipzig vgl. Wendt 2018, zur Geschichte linker Aneignungen der ,Provinz' in Westdeutschland Siegfried 2006. Einen interessanten Spezialfall stellt Chemnitz dar, das als - wahrnehmungsmäßig oft ,vergessene ${ }^{\text {‘ }}$ und verkehrstechnisch schlecht angebundene - drittgrößte ostdeutsche Stadt einerseits bezüglich Leerstand etc. viele jener Merkmale eines ,Experimentierfeldes‘ aufweist, die in Leipzig vor zehn Jahren deutlicher als heute vorhanden waren, wo andererseits aber eine entsprechende zivilgesellschaftliche Grundierung schmerzlich vermisst wird, vgl. dazu den Beitrag von Dominik Intelmann in diesem Heft.

[12] Vgl. auch das Zukunftsszenario „Integralland“ in Böttger/Carsten/Engel 2016: 103ff.

[13] Eine solche Begrifflichkeit könnte auch das zuletzt wieder häufiger debattierte Konzept ,Heimat' je nachdem hinter sich lassen oder radikal neu begründen.

\section{Autor_innen}

Tobias Bernet ist Historiker und Ethnologe und forscht zu Stadtentwicklung, Wohnungspolitik und sozialen Bewegungen.

tobiasbernet@zedat.fu-berlin.de

\section{Literatur}

Asch, Ronald (2018): Das Selbstbild der Unbelehrten. In: Frankfurter Allgemeine Zeitung, 3.1.2018, 9 .

Bangel, Christian (2018): Die Brücke. In:Zeit online, 17.3.2018. https://www.zeit.de/politik/ deutschland/2018-03/oberbuergermeisterwahl-frankfurt-oder-rene-wilke-martin-wilkearbeitslosigkeit-rassismus-generationskonflikt (letzter Zugriff am 16.4.2019). 
Barber, Benjamin (1992): Jihad vs. McWorld. In: The Atlantic 3/1992, 53-65.

Barber, Benjmanin (2013): If Mayors Ruled the World: Dysfunctional Nations, Rising Cities. New Haven: Yale University Press.

Barber, Benjmanin (2016): Can cities counter the power of President-elect Donald Trump? https://www.thenation.com/article/can-cities-counter-the-power-of-president-electdonald-trump/ (letzter Zugriff am 10.10.2018).

Barstow, David / Craig, Susanne / Buettner, Russ (2018): Trump engaged in suspect tax schemes as he reaped riches from his father. In: The New York Times online, 02.10.2018. https://www.nytimes.com/interactive/2018/10/02/us/politics/donald-trump-taxschemes-fred-trump.html (letzter Zugriff am 10.10.2018).

Begrich, David (2018): Liebe westdeutsche Freund/innen. http://telegraph.cc/liebewestdeutsche-freund-innen/ (letzter Zugriff am 10.10.2018).

Benicke, Jens (2016): Autorität und Charakter. Wiesbaden: Springer VS.

Bergmann, Knut / Diermeier, Matthias / Niehues, Judith (2018): Ein komplexes Gebilde: Eine sozio-ökonomische Analyse des Ergebnisses der AfD bei der Bundestagswahl 2017. In: Zeitschrift für Parlamentsfragen 49/2, 243-264.

Bishop, Bill / Cushing, Robert (2008): The Big Sort: Why the Clustering of Like-Minded America is Tearing Us Apart. Boston: Houghton Mifflin.

Böttger, Matthias / Carsten, Stefan / Engel, Ludwig (Hg.) (2016): Spekulationen Transformationen: Überlegungen zur Zukunft von Deutschlands Städten und Regionen. Zürich: Lars Müller Publishers.

Bozic, Ivo (2016): Bye-bye, Westen. In: Jungle World, 17.11.2016, 3.

Brandau, Bastian (2017): An den Rand gedrängt. http://www.deutschlandfunk.de/niedriglohnland-erzgebirge-an-den-rand-gedraengt.1769.de.html?dram:article_id=395656 (letzter Zugriff am 10.10.2018).

Brechtken, Magnus (2017): Das Selbstbild der Unberührbaren. In: Frankfurter Allgemeine Zeitung, 18.12.2017, 12.

Brenner, Neil (2014): Implosions / Explosions: Towards a Study of Planetary Urbanization. Berlin: Jovis.

Cantoni, Davide / Hagemeister, Felix / Westcott, Mark (2019): Persistence and Activation of Right-Wing Political Ideology. SFB Rationality \& Competition, Discussion Paper 143. München: Ludwig-Maximilians-Universität.

Cassee, Andreas (2016): Globale Bewegungsfreiheit: Ein philosophisches Plädoyer für offene Grenzen. Berlin: Suhrkamp.

Diener, Roger / Herzog, Jacques / Meili, Marcel / de Meuron, Pierre / Schmid, Christian (2005): Die Schweiz - ein städtebauliches Portrait. Basel: Birkhäuser.

Dörre, Klaus (2018): In der Warteschlange: Rassismus, völkischer Populismus und die Arbeiterfrage. In: Karina Becker / Klaus Dörre / Peter Reif-Spirek (Hg.), Arbeiterbewegung von rechts? Ungleichheit, Verteilungskämpfe, populistische Revolte. Frankfurt am Main: Campus, 49-80.

Dowling, Emma / van Dyk, Silke / Graefe, Stefanie (2017): Rückkehr des Hauptwiderspruchs? Anmerkungen zur aktuellen Debatte um den Erfolg der Neuen Rechten und das Versagen der „Identitätspolitik“. In: Prokla 47/3, 411-420.

Eckardt, Frank / Seyfarth, René / Werner, Franziska (Hg.) (2015): Leipzig: Die neue urbane Ordnung der unsichtbaren Stadt. Münster: Unrast.

Eisenring, Christoph (2016): In Deutschlands „Schwarmstädten“. In: Neue Zürcher Zeitung online, 8.8.2016. https://www.nzz.ch/wirtschaft/wirtschaftspolitik/wo-es-jungemenschen-hinzieht-in-deutschlands-schwarmstaedten-ld.109558 (letzter Zugriff am 10.10.2018).

Isin, Engin F. (2002): Being Political: Genealogies of Citizenship. Minneapolis: University of Minnesota Press.

Feddersen, Jan (2017): Anschwellender Albtraum. In: taz, 25.9.2017, 5.

Florida, Richard (2017a): The New Urban Crisis: How Our Cities Are Increasing Inequality, Deepening Segregation, and Failing the Middle Class and What We Can Do About It. New York: Basic Books.

Florida, Richard (2017b): The United Cities of America. https://www.citylab.com/ equity/2017/04/the-united-cities-of-america/520221/ (letzter Zugriff am 10.10.2018).

Foroutan, Naika / Kubiak, Daniel (2018): Ausgeschlossen und abgewertet: Muslime und Ostdeutsche. In: Blätter für deutsche und internationale Politik 63/7, 94-102. 
Frank, Thomas (2004): What's the Matter with Kansas? How Conservatives Won the Heart of America. New York: Henry Holt \& Co.

Franz, Christian / Fratzscher, Marcel / Kritikos, Alexander (2018): AfD in dünn besiedelten Räumen mit Überalterungsproblemen stärker. In: DIW Wochenbericht 8/2018, 136-144.

Gasser, Florian / Hamann, Götz / Pausackl, Christina / Rohrbeck, Felix / Schulz, Bettina (2016): Rettet die Provinz! In: Die Zeit, 1.9.2016, 19.

Glaeser, Edward (2011): Triumph of the City: How Our Greatest Invention Makes Us Richer, Smarter, Greener, Healthier, and Happier. New York: Penguin.

Goebel, Michael (2016): Liberal Cities? What Recent Elections Mean for Global Urban History. https://globalurbanhistory.com/2016/12/29/liberal-cities-what-recentelections-mean-for-global-urban-history/ (letzter Zugriff am 10.10.2018).

Graham, David (2017): Red State, Blue City. In: The Atlantic 3/2017, 24-26.

Groß, Eva / Zick, Andreas / Krause, Daniela (2012): Von der Ungleichwertigkeit zur Ungleichheit: Gruppenbezogene Menschenfeindlichkeit. In: Aus Politik und Zeitgeschichte 62/16-17, 11-18.

Hardt, Michael / Negri, Antonio (2009): Commonwealth. Cambridge: Harvard University Press.

Haupt, Heinz-Gerhard (2004): Guild Theory and Guild Organization in France and Germany During the Nineteenth Century. In: Mark Bevir / Frank Trentmann (Hg.), Markets in Historical Contexts: Ideas and Politics in the Modern World. Cambridge: Cambridge University Press, 90-104.

Hauser, Kornelia (2017): Die Tea-Party in Selbstbehauptungskämpfen: Rezension zu Arlie Russell Hochschild, Strangers in Their Own Land. https://www.kritisch-lesen.de/ rezension/die-tea-party-in-selbstbehauptungskampfen (letzter Zugriff am 10.10.2018).

Hawkins, Mike (1994): Durkheim on Occupational Corporations: An Exegesis and Interpretation. In: Journal of the History of Ideas 55/3, 461-481.

Hensel, Jana (2017): Aus Wut folgt Wut folgt Wut. In: Die Zeit, 24.8.2017.

Herbert, Ulrich (2002): Liberalisierung als Lernprozess: Die Bundesrepublikin der deutschen Geschichte - eine Skizze. In: Ders. (Hg.): Wandlungsprozesse in Westdeutschland. Göttingen: Wallstein, 7-49.

Hess, Sabine / Lebuhn, Henrik (2014): Politiken der Bürgerschaft: Zur Forschungsdebatte um Migration, Stadt und Citizenship. In: sub\urban 2/3, 11-34.

Hillje, Johannes (2018): Rückkehr zu den politisch Verlassenen: Gespräche in rechtspopulistischen Hochburgen in Deutschland und Frankreich. Berlin: Das Progressive Zentrum.

Hochschild, Arlie Russell (2017): Fremd in ihrem Land: Eine Reise ins Herz der amerikanischen Rechten. Frankfurt am Main: Campus.

Hotz, Stefan (2018): Das Ringen um die Agglomeration. In: Neue Zürcher Zeitung, 24.4.2018, 14 .

Jacobs, Jane (1961): The Death and Life of Great American Cities. New York: Random House.

Kaschuba, Wolfgang (2016): Die Rache der Dörfer (Interview). https://www.deutschlandfunkkultur.de/rechtspopulismus-die-rache-der-doerfer.1008.de.html?dram:article_ id=371289 (letzter Zugriff am 10.10.2018).

Kleffner, Heike / Meisner, Matthias (2017): Unter Sachsen: Zwischen Wut und Willkommen. Berlin: Ch. Links.

Koester, Elsa (2018): Hase, du bleibst hier. In: Der Freitag, 13.9.2018. https://www.freitag. de/autoren/elsa-koester/hase-du-bleibst-hier (letzter Zugriff am 10.10.2018).

Körner, Vincent (2018): Eine Sache der geschäftlichen Interessen: Der Immobiliensektor ist hoch anfällig für Geldwäsche. https://oxiblog.de/eine-sache-der-geschaeftlicheninteressen-geldwaesche-immobiliensektor/ (letzter Zugriff am 10.10.2018).

Koppetsch, Cornelia (2017): Aufstand der Etablierten? Rechtspopulismus und die gefährdete Mitte. https://soziopolis.de/beobachten/kultur/artikel/aufstand-der-etablierten/ (letzter Zugriff am 10.10.2018).

Köpping, Petra (2018): Integriert doch erst mal uns! Eine Streitschrift für den Osten. Berlin: Ch. Links.

Koseki, Shin Alexandre (2017): Moral Matrices: Space Through the Unfolding of Direct Democracy in Switzerland (Dissertation). Lausanne: EPFL.

Kuper, Simon (2017): Wüsten ohne Bistro, Bus und Arzt. In: Neue Zürcher Zeitung online, 22.5.2017. https://www.nzz.ch/meinung/kommentare/stadt-und-land-die-doerfer-neubeleben-ld.1294244 (letzter Zugriff am 10.10.2018). 
Leggewie, Claus (2017): Die Abgehängten: Zur Debatte über soziale Ungerechtigkeit und deren Terminologie. https://ia902906.us.archive.org/11/items/LeggewieAbgehaengten/ Leggewie_Abgehaengte_NDR_Gedankenzurzeit_170806.pdf (letzter Zugriff am 6.4.2019).

Lengfeld, Holger (2017a): Die „Alternative für Deutschland“: Eine Partei für Modernisierungsverlierer? In: Kölner Zeitschrift für Soziologie und Sozialpsychologie 69/2, 209-232.

Lengfeld, Holger (2017b): AfD-Wähler sind nicht wirtschaftlich, sondern kulturell abgehängt (Interview mit Ruth Schneeberger). In: Sueddeutsche Zeitung online, 2.9.2017. https:// www.sueddeutsche.de/kultur/abgehaengte-bevoelkerungsgruppen-afd-waehler-sindnicht-wirtschaftlich-sondern-kulturell-abgehaengt-1.3675805 (letzter Zugriff am 10.10.2018).

Lessenich, Stephan (2018): Der Klassenkampf der Mitte. In: Süddeutsche Zeitung, 3.1.2018, 9 .

Müller, Henrik (2018): Landluft macht unfrei. In: Spiegel online, 5.8.2018. http:// www.spiegel.de/wirtschaft/soziales/landflucht-die-abwanderung-schadet-derdemokratie-a-1221687.html (letzter Zugriff am 10.10.2018).

Münter, Angelika / Osterhage, Frank (2018): Trend Reurbanisierung? Analyse der Binnenwanderungen in Deutschland 2006 bis 2015. Gütersloh: Bertelsmann-Stiftung.

Nassehi, Armin (2015): Die letzte Stunde der Wahrheit: Warum rechts und links keine Alternativen mehr sind und Gesellschaft ganz anders beschrieben werden muss. Hamburg: Murmann.

Nassehi, Armin (2016): Nicht nur die Rechten: Warum die Moderne so anstrengend ist. In: Kursbuch 186, 59-82.

Priemer, Jana / Krimmer, Holger / Labigne, Anaël (2018): Vielfalt verstehen, Zusammenhalt stärken: ZiviZ-Survey 2017. Essen: Stifterverband.

Pro Asyl (2017): Gewalt gegen Flüchtlinge 2017: Brandenburg und Sachsen traurige Spitzenreiter. https://www.proasyl.de/pressemitteilung/gewalt-gegen-fluechtlinge-2017brandenburg-und-sachsen-traurige-spitzenreiter-auch-bundesweit-kein-grund-zurentwarnung/ (letzter Zugriff am 10.10.2018).

Putnam, Robert (mit Robert Leonardi und Raffaella Y. Nanetti) (1993): Making Democracy Work: Civic Traditions in Modern Italy. Princeton: Princeton University Press.

Rada, Uwe (2017): Die Rache der Peripherie. In: taz, 30.9.2017, 11.

Reckwitz, Andreas (2016): Zwischen Hyperkultur und Kulturessenzialismus: Die Spätmoderne im Widerstreit zweier Kulturalisierungsregimes. https://soziopolis.de/beobachten/ kultur/artikel/zwischen-hyperkultur-und-kulturessenzialismus/ (letzter Zugriff am 10.10.2018).

Reckwitz, Andreas (2017): Die Gesellschaft der Singularitäten: Zum Strukturwandel der Moderne. Berlin: Suhrkamp.

Reckwitz, Andreas (2018): Das Band zerreißt. In: Die Zeit, 22.2.2018, 43.

Rennefanz, Sabine (2018): Kommentar zu Chemnitz: Die Wurzeln der Wut reichen viel weiter zurück. In: Berliner Zeitung online, 31.08.2018. https://www.berliner-zeitung. $\mathrm{de} /$ politik/meinung/kommentar-zu-chemnitz-die-wurzeln-der-wut-reichen-viel-weiterzurueck--31197046 (letzter Zugriff am 10.10.2018).

Rink, Dieter / Schönig, Barbara / Gardemin, Daniel / Holm, Andrej (2015): Städte unter Druck: Die Rückkehr der Wohnungsfrage. In: Blätter für deutsche und internationale Politik 6o/6, 69-79.

Rosa, Hartmut (2016): Resonanz: Eine Soziologie der Weltbeziehung. Berlin: Suhrkamp.

Rossi, Ugo (2018): The Populist Eruption and the Urban Question. In: Urban Geography 39/9, 1425-1430.

Sandifer, Phil (2016): Theses on Trump 16. https://archive.is/E3c68 (letzter Zugriff am 10.10.2018).

Saunders, Doug (2011): Arrival City: How the Largest Migration in History is Reshaping Our World. London: Windmill Books.

Schäfer, Armin (2017): Kultur statt Ökonomie. In: Frankfurter Allgemeine Zeitung, 16.10.2017, 6 .

Schaible, Jonas (2018): Wieso es keinen Rechtsruck gibt, aber die extreme Rechte trotzdem wächst. https://www.t-online.de/nachrichten/deutschland/gesellschaft/id_84519312/ wieso-es-keinen-rechtsruck-gibt-aber-die-extreme-rechte-trotzdem-waechst.html (letzter Zugriff am 22.3.2019) 
Scherr, Albert / Hofmann Rebecca (2016): Sanctuary Cities: Eine Perspektive für deutsche Kommunalpolitik? In: Kritische Justiz 49/1, 86-97.

Schulz, Daniel (2018): Wir waren wie Brüder. In: taz, 29.9.2018.

Schulz, Jörn (2019): Gegen Wurzeln und Vaterländerei. In: Jungle World, 10.1.2019, 3.

Siegfried, Detlef (2006): Urbane Revolten, befreite Zonen: Über die Wiederbelebung der Stadt und die Neuaneignung der Provinz durch die „Gegenkultur“ der 1970er Jahre. In: Adelheid von Saldern (Hg.): Stadt und Kommunikation in bundesrepublikanischen Umbruchszeiten. Stuttgart: Franz Steiner, 351-366.

Silver, Nate (2016): Education, Not Income, Predicted Who Would Vote For Trump. https:// fivethirtyeight.com/features/education-not-income-predicted-who-would-vote-fortrump/ (letzter Zugriff am 10.10.2018).

Simons, Harald / Weiden, Lukas (2015): Schwarmstädte in Deutschland: Ursachen und Nachhaltigkeit der neuen Wanderungsmuster (Endbericht). Berlin: GdW.

Simons, Harald / Weiden, Lukas (2016): Schwarmverhalten in Sachsen: Umfang, Ursache, Nachhaltigkeit und Folgen der neuen Wanderungsmuster. Dresden: VDW Sachsen.

Stokowski, Margarete (2019): Warum „linksgrün versifft“? In: Spiegel online, 12.2.2019. http://www.spiegel.de/kultur/gesellschaft/rechte-sprache-warum-linksgruenversifft-a-1252819.html (letzter Zugriff am 21.3.2019).

Tutt, Cordula (2017): Ganze Landstriche verlieren junge Menschen. https://www.wiwo. de/politik/deutschland/landflucht-der-jugend-ganze-landstriche-verlieren-jungemenschen/20478294.html (letzter Zugriff am 10.10.2018).

Vehrkamp, Robert / Wegschaider, Klaudia (2017): Populäre Wahlen: Mobilisierung und Gegenmobilisierung der sozialen Milieus bei der Bundestagswahl 2017. Gütersloh: Bertelsmann-Stiftung.

Volk, Pia (2013): Kann Leipzig Hypezig überleben? In: ZEIT online, 01.10.2013. https:// www.zeit.de/lebensart/2013-10/leipzig-hypezig-gentrifizierung-wohnkonzepte-stadtentwicklung/komplettansicht (letzter Zugriff am 10.10.2018).

Wendt, Matthias (2018): „Weil es nur zusammen geht“: Commons-basierte Selbstorganisation in der Leipziger Hausprojekteszene. Frankfurt am Main: Campus.

Wieseke, Dirk (2017): Wie ticken AfD-Sympathisanten? http://www.horizont.net/ planung-analyse/nachrichten/Online-Special-Wahlforschung-Wie-ticken-AfD-Sympathisanten-159673 (letzter Zugriff am 10.10.2018).

Woodard, Collin (2011): American Nations: A History of the Eleven Rival Regional Cultures of North America. New York: Viking.

Zelik, Raul / Bruchmann, Hanno / Candeias, Mario (2016): Rebellische Städte: Erfolg oder Frust? In: Luxemburg 2/2016, 98-107.

Zürn, Michael (2016): Jenseits der Klassenfrage: Neue Konfliktlinien zeigen sich in Europa, der Türkei und Amerika. In: WZB-Mitteilungen 154, 7-10.

\section{City States or Barbarism? (Anti-)Urbanism, Demographics, and Municipal Perspectives}

This essay examines the current popularity of right-wing politics from the perspective of critical urban studies. Focusing on demographic processes, particularly internal migration, it discusses the thesis that rightist ideas and groups enjoy higher approval in, left-behind'rural areas while finding less resonance among urban populations considered more, cosmopolitan and approving of modernization. Considering both German and American debates, the text argues that the spatialization of political attitudes should be conceptualized in a more dynamic and relational manner, putting emphasis on small-scale differences within cities and regions. This points towards the emancipatory potential of the municipal as a level of political decision making beyond the nation state. 